\title{
Matching Highly Non-ideal Ocular Images: An Information Fusion Approach *
}

\author{
Arun Ross, Raghavender Jillela (West Virginia University) \\ Jonathon M. Smereka, Vishnu Naresh Boddeti, B. V. K. Vijaya Kumar (Carnegie Mellon University) \\ Ryan Barnard, Xiaofei Hu, Paul Pauca, Robert Plemmons (Wake Forest University)
}

\begin{abstract}
We consider the problem of matching highly non-ideal ocular images where the iris information cannot be reliably used. Such images are characterized by non-uniform illumination, motion and de-focus blur, off-axis gaze, and non-linear deformations. To handle these variations, a single feature extraction and matching scheme is not sufficient. Therefore, we propose an information fusion framework where three distinct feature extraction and matching schemes are utilized in order to handle the significant variability in the input ocular images. The Gradient Orientation Histogram $(\mathrm{GOH})$ scheme extracts the global information in the image; the modified Scale Invariant Feature Transform (SIFT) extracts local edge anomalies in the image; and a Probabilistic Deformation Model (PDM) handles nonlinear deformations observed in image pairs. The simple sum rule is used to combine the match scores generated by the three schemes. Experiments on the extremely challenging Face and Ocular Challenge Series (FOCS) database and a subset of the Face Recognition Grand Challenge (FRGC) database confirm the efficacy of the proposed approach to perform ocular recognition.
\end{abstract}

\section{Introduction}

The use of the ocular region as a biometric trait has gained considerable traction in recent years. Besides the eye and the structures within the eye (viz., iris, retina, and sclera), the ocular region includes the eyelids, eyelashes, eyebrow and the skin texture in the vicinity of the eye. Traditionally, research in ocular biometrics has focused on segmenting and processing the iris texture primarily due to its

\footnotetext{
* This work was sponsored under IARPA BAA 09-02 through the Army Research Laboratory and was accomplished under Cooperative Agreement Number W911NF-10-2-0013. The views and conclusions contained in this document are those of the authors and should not be interpreted as representing official policies, either expressed or implied, of IARPA, the Army Research Laboratory, or the U.S. Government. The U.S. Government is authorized to reproduce and distribute reprints for Government purposes notwithstanding any copyright notation herein. Corresponding author: Arun Ross (Arun.Ross@mail.wvu.edu)
}

high matching accuracy [6]. However, this accuracy is predicated on the quality of the input ocular image and its spatial resolution. When the quality of the input image deteriorates or when the stand-off distance between the eye and the camera increases, the matching accuracy due to the iris biometric can significantly degrade. This has led to an increased interest in utilizing the region surrounding the iris for enhancing the accuracy of the biometric system.

In this context, the use of the periocular region as a biometric cue has been investigated. Periocular biometric, as defined in [20], specifically refers to the externally visible skin region of the face that surrounds the eye socket. The utility of this trait is especially pronounced when the iris is occluded (e.g., due to eyelid closure) or when the iris cannot be accurately segmented (e.g., due to low image quality). While earlier work on periocular biometrics focused on images acquired in the visible spectrum [14, 11, 13], more recent work has explored the processing of images in the near-infrared spectrum [20]. From a practical application standpoint, the impact of acquisition distance on periocular matching performance has been studied [2].

In this work, we seek to answer the following question: Is it possible to perform biometric recognition using highly non-ideal ocular data? This type of data is commonly encountered in unconstrained environments (e.g., iris-on-themove type of systems) where ocular images exhibit nonuniform illumination, severe blur, occlusion, oblique gaze, etc. Further, the dimensions of the eye region can significantly vary across images. See Figure 1. In other words, the intra-class variations observed in such images can be significantly large. Our goal is to design effective mechanisms for performing feature extraction and matching on this data. In particular, we hypothesize that an information fusion approach is necessary to process this data.

To this end, we present a biometric fusion framework for processing and matching ocular images acquired under highly non-ideal conditions. The main contributions of this work are as follows:

1. We highlight the various types of challenges associated with ocular recognition when using images that are acquired from moving subjects in an unconstrained envi- 


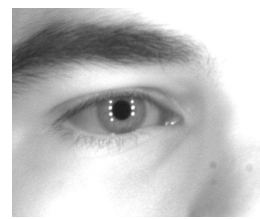

(a)

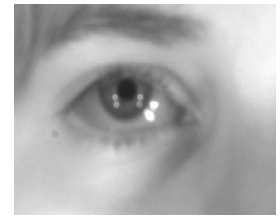

(g)

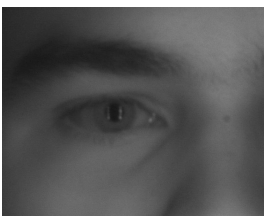

(b)

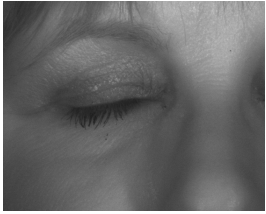

(h)

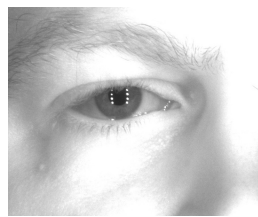

(c)

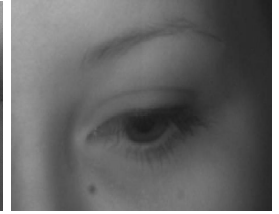

(i)

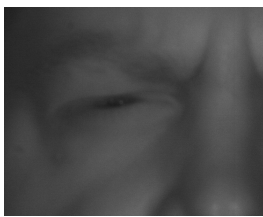

(d)

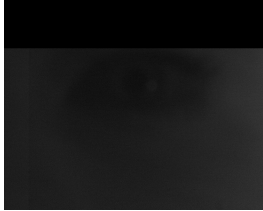

(j)

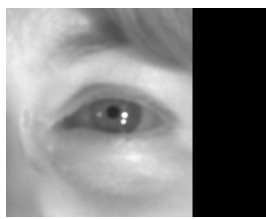

(e)

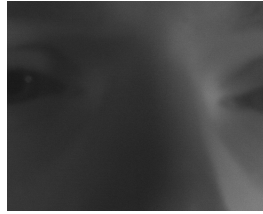

(k)

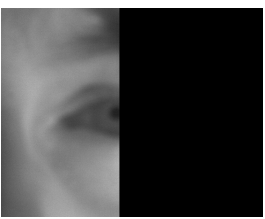

(f)

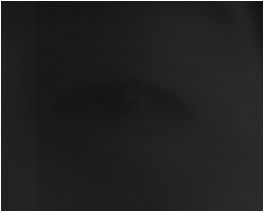

(1)

Figure 1. Non-ideal ocular images demonstrating some of the challenges addressed in this work.

ronment.

2. We describe an information fusion approach to improve the performance of ocular recognition in this very challenging imagery.

3. We demonstrate the advantage of using the entire ocular region, rather than the iris information alone, in cases where the iris may be occluded or when the quality of the acquired images is very low.

\section{Challenging Ocular Images: An Overview}

The ocular image subset from the Face and Ocular Challenge Series (FOCS) database [12] was used in this work. The FOCS database was collected primarily to study the possibility of performing ocular (iris and periocular) recognition in images obtained under severely non-ideal conditions. Ocular images of dimension $750 \times 600$ pixels were captured from subjects walking through a portal, to which a set of Near Infra Red (NIR) sensors and illuminators were affixed. Since no constraints were imposed on the walking subjects, a large number of the acquired images are of very poor quality. The images from the FOCS ocular subset are known to be extremely challenging for the task of biometric recognition, mainly due to the following reasons:

1. High levels of illumination variation: The level of illumination observed across a set of images varies significantly in this database. This is caused by the variation in the stand-off distance, which is in turn caused by subject motion. Figure 1 (a) and (b) show two images of a single subject exhibiting high levels of illumination variation.

2. Deformation around the eye region: Many images in the FOCS database exhibit non-rigid, inconsistent deformations of the eyebrows, eyelids, and the skin region. These deformations could be caused by involuntary blinking or closing action of the eye. In such images, the iris is partially or completely occluded, proving to be a major challenge in performing both periocular and iris recognition. Figure 1 (c) and (d) show a pair of images corresponding to a single subject exhibiting such deformation.

3. Padding of the images: Due to the unconstrained image acquisition setup, the size of many periocular images were smaller than $750 \times 600$ pixels. Such images were padded with pixels of zero intensity (by the distributors of the database) in order to maintain a constant image size, as seen in Figure 1 (e) and (f).

4. Extremely poor quality images: The quality of some of the images in the FOCS database is extremely poor due to blur, occlusions, and gaze deviation as seen in Figure 2 (g), (h), and (i), respectively. Additionally, in some images, it is difficult even for a human expert to determine the location of the physical features (eye, eyebrow, etc.). Some examples are shown in Figure $1(\mathrm{j}),(\mathrm{k})$ and (l).

5. Intra-class variability and inter-class similarity: The images in this database were observed to exhibit high intra-class variability and inter-class similarity, again caused by the motion of the subjects and significant variation in illumination. Example images are shown in Figure 2.

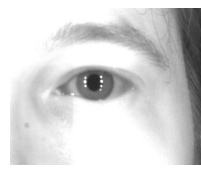

(a)

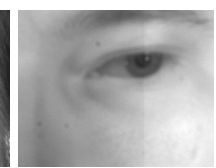

(b)

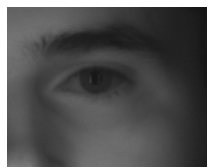

(c)

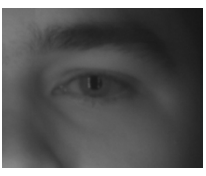

(d)
Figure 2. (a) and (b): Image pair corresponding to a single subject, exhibiting intra-class variability. (c) and (d): Images corresponding to two different subjects exhibiting inter-class similarity.

\section{Motivation and Proposed Approach}

The iris biometric is not expected to provide good recognition performance on images in the FOCS database (this is later demonstrated in Section 5), mainly due to two reasons: (a) difficulty in segmenting the iris, and (b) lack of suffi- 
cient information in the iris region, even if it is segmented successfully. Therefore, using the entire ocular region appears to be the best alternative for biometric recognition on this imagery. However, even though the challenges listed in Section 2 render the task of performing ocular recognition quite challenging and unique.

Many feature extraction techniques have been proposed in the existing literature to perform periocular recognition. Depending on the region of interest from which the features are extracted, a majority of the existing periocular techniques can be classified into two categories: global (e.g., GOH [14], GIST [2], etc.) or local (e.g., SIFT [14, 15]; also see [11]). While global feature extractors summarize features from the entire image (e.g., shape, color, texture, etc.), local feature extractors gather information around a set of detected key points. Existing studies report reasonably good periocular recognition performance when using just one type of feature extraction scheme. Such performances can be mainly attributed to the high quality of the input images. Intuitively, such an approach may not be suitable for the FOCS database, owing to the differing quality of images across the database. Furthermore, a robust scheme is required to handle the non-rigid deformations occurring in the periocular region. To this end, the current work adopts an information fusion technique to improve ocular recognition performance. The major aspects of the proposed technique are: (i) to summarize the discriminant information from the entire image, a global feature extraction and matching scheme is implemented using Gradient Orientation Histograms (GOH) [5], (ii) to handle scale and shift irregularities, local information is obtained using a modified version of the Scale Invariant Feature Transformation (SIFT) [8], and (iii) to handle the non-rigid and inconsistent deformations of the eyebrows and eyelids within the periocular region, Probabilistic Deformation Models (PDM) [17] are used. This method determines the similarity between corresponding sub-patches of two images, rather than utilizing the global or local features.

The discriminant information extracted using the above three techniques could be agglomerated at various levels (e.g., feature level, score level, etc.). For brevity purposes, and to avoid training, the proposed approach fuses the biometric information at the score level using a simple sum rule.

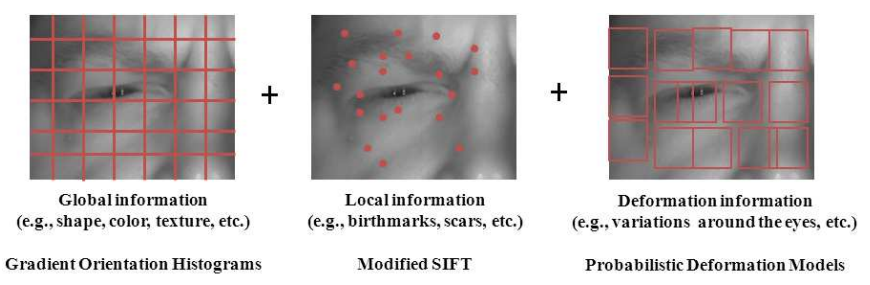

Figure 3. The three methods used for processing non-ideal ocular images.

\section{Feature Extraction}

The pre-processing and implementation details for each of the feature extraction schemes used in this approach are discussed in this section.

\subsection{Gradient Orientation Histograms (GOH)}

Given an ocular image, the Gradient Orientation Histograms approach is used to extract global information from a defined region of interest. Defining a region of interest helps in (a) maintaining uniformity when matching corresponding regions of two separate images, and (b) generating feature vectors of fixed sizes, which facilitates a quick comparison between two given images. To alleviate the problem of drastic variations in the lighting and to increase its contrast, illumination normalization was performed prior to extracting the features.

An eye center detector [19] was used to facilitate geometric normalization of a given image and to determine a fixed region of interest for feature extraction. The output of this process is the $2 \mathrm{D}$ location of the center of the iris in a given image. The eye center detector is based on the shift-invariance property of the correlation filters. The correlation filter for the eye center detector was trained on a set of 1000 images of the FOCS database, in which the eye centers were manually labeled. When the correlation filter is applied to an input image, a relatively sharp peak is observed in the correlation output, whose location corresponds to the center of the eye. The detection accuracy of the eye detector was observed to be $95 \%$. The output of the detector was used as an anchor point to crop a region of size $500 \times 400$ pixels. Feature extraction is then performed at specific points, that are sampled at an interval of 5 pixels in the $\mathrm{x}$ - and $\mathrm{y}$-directions over the image. The gradient orientation information of the pixels lying within a specified region around a sampling point is then accumulated into an eight bin histogram. The gradient information at a pixel $(x, y)$, for an image $I$, is computed using the expression $\arctan \left(\frac{I(x, y+1)-I(x, y-1)}{I(x+1, y)-I(x-1, y)}\right)$. The histograms corresponding to all the sampling points are then concatenated to form the feature vector of size $(1 \times 64,000)$. Finally, matching between two images is performed by considering the Euclidean distance between their corresponding feature vectors.

\subsection{Modified Scale Invariant Feature Transform}

For applications with relaxed imaging constraint scenarios, rotation of the face or eye gaze can significantly affect the $2 \mathrm{D}$ representation of ocular features, as seen in Section 2. The relative invariance of the Scale Invariant Feature Transform (SIFT) [7], to translation, scale, and orientation change makes SIFT a potentially viable and robust method for ocular recognition. Given a periocular image, 
the SIFT algorithm produces a set of keypoints and feature vectors describing various invariant features found in the image. The match score between two images $f_{1}$ and $f_{2}$ is obtained by comparing their corresponding keypoints, and counting the number of keypoints that match with each other. A key advantage of this simple approach for ocular region matching is that it avoids detection and segmentation of the iris or other anatomical features, which is often challenging. Moreover, the relative invariance of SIFT keypoints to scale and spatial shift reduces the need for accurate registration of the ocular regions being compared.

Although SIFT has been successfully used to perform face [9] and iris recognition [1], direct application of SIFT on challenging datasets, such as the FOCS dataset, may result in a poor overall performance [15]. This is primarily due to a large number of false matches during the the matching process, caused by the drastic variations in the illumination and blur. To alleviate these problems on challenging periocular imagery, the application of SIFT is modified in the following way:

1. Image pre-processing: To improve computational efficiency, the input ocular images are resized to have 480 pixels in height using bicubic interpolation, while the width is varied to preserve the original aspect ratio. In addition, adaptive local histogram equalization is performed to improve the image contrast before the SIFT algorithm is applied.

2. Feature encoding: An open source implementation of the SIFT algorithm provided in the VLFeat library [18] was used in this work. The parameter space was explored to maximize the ratio of matching performance to computation time. In particular, it was observed that the peak threshold parameter had the largest effect on both the performance and the computation time. Empirical evaluations suggested an optimal value of 2 for this parameter. After incorporating the specified changes, feature encoding was performed on the entire pre-processed image, without additional scaling or registration.

3. Feature matching: Additional space-based constraints were incorporated in the matching stage, to improve the accuracy of matched keypoints. Specifically, in order for a pair of keypoints matched by the SIFT algorithm to be accepted as a true match they must satisfy the following two conditions: (a) Proximity constraint: The keypoints should be located in approximately the same image region; (b) Orientation constraint: The gradient direction orientations [7] of the keypoints should be sufficiently similar. Thresholds for the keypoint proximity and orientation are adjusted based on the application. In this work, these thresholds are set to a value of $35 \%$ of the image height for proximity, and 20 degrees for orientation.

Figure 4.2(a) illustrates the result obtained by matching keypoints using the standard implementation of the SIFT algorithm. It can be observed that many keypoints in disparate physical regions are matched together. This is due to the similarities found in certain features such as hair in the eyebrow and skin patches. The improvement obtained by incorporating the additional constraints on the proximity and orientation of matched SIFT keypoints can be observed in Figure 4.2(b). The addition of these constraints leads to improved matching, thereby enhancing the overall recognition performance using challenging periocular imagery.

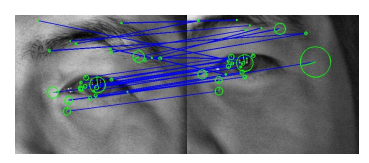

(a)

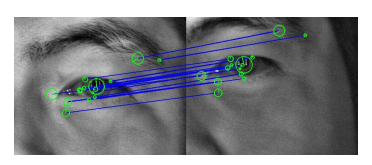

(b)
Figure 4. (a): Keypoints matched by the standard implementation of SIFT. (b): Matching keypoints obtained after applying the additional constraints on the proximity and the orientation parameters. Notice that the proposed constraints help in discarding spurious matches between keypoints.

\subsection{Probabilistic Deformation Models (PDM)}

As mentioned in Section 2, handling the non-rigid deformation between two periocular images of FOCS database is very critical in achieving a good recognition performance. For this purpose, the probabilistic matching algorithm, adopted from the technique originally proposed by Thornton et al. [17] for iris matching, is used. Given two images (a probe and a gallery) this process produces a similarity score by taking into account the relative nonstationary distortion occurring between them. The measure of deformation and similarity is obtained by segmenting the probe image into non-overlapping patches (to provide tolerance to valid local distortions), and correlating each patch with the corresponding template patch. The final match score is based on the individual patch match scores and the corresponding deformation estimate from the respective correlation planes. The main premise behind this algorithm is that, for a genuine match between the template and probe, besides a high match score, the deformation pattern from the patches must also be valid for that class of patterns.

The fusion Optimal Trade-off Synthetic Discriminant Function (OTSDF) correlation filter [17], based on the standard OTSDF filter [16], is used for the template design. The template is specifically designed to produce a sharp peak at the center of the correlation plane for a genuine match, and no such peak for an impostor match. A deformation is said to occur when the correlation peak is shifted from the center of the image region as seen in Figure 5. For a given pattern class (i.e, ocular region of a subject), the set of valid distortions is learned from the training data. To effectively learn and distinguish a valid distortion from just random 

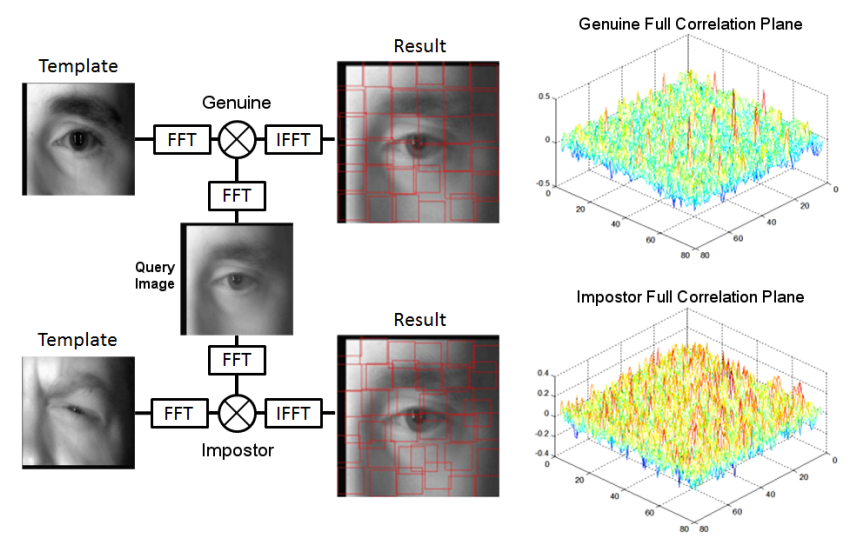

Figure 5. An example of genuine and impostor matching using the PDM approach. The red boxes indicate each patch that is correlated against the template. The boxes are centered on the highest correlation value in the correlation plane in order to display the shifts that occur. The shifts seem to be correlated when matching the probe image with the genuine template; however, when comparing the probe with the impostor template, the shifts are seemingly random.

movements, an undirected graph is used to capture the correlation between the shifts (rather than causation), thereby approximating the true match. The final score is computed by performing a maximum-a-posteriori (MAP) estimation on the probability that a probe image matches a template image given some deformation in the image plane.

\subsubsection{OTSDF Filter}

It is particularly difficult to design a single robust correlation filter $(\mathrm{CF})$ that is tolerant to intra-class distortions that can occur in the ocular regions (e.g., closed eye lids, raised eyebrows, occlusions, etc.). However, there is an increased chance of obtaining higher overall similarity values for genuine image pairs by designing several CFs per region. Therefore, the fusion OTSDF correlation filter is used in this work to jointly satisfy the design criteria via multiple degrees of freedom, leading to robust discrimination for detecting similarities between images of the same subject. In contrast to the individual OTSDF filter design, the fusion CF design takes advantage of the joint properties of different feature channels to produce the optimal output plane. Each channel produces a similarity metric based on a relative transformation of the observed pattern and the inner sum represents a spatial cross-correlation between the channels giving an increased chance that the similarity metric will produce high peaks for genuine image pairs.

\subsubsection{MAP Estimation}

The goal of this technique is to authenticate a genuine image by a template, and reject an impostor image. CFs pro- vide a reliable approach to obtaining a similarity measure between a template and probe image. However, in the presence of deformations, the matching performance of CFs may deteriorate. Even after independently correlating nonoverlapping regions of each image, a good measure of similarity between the probe and template needs to account for distortions (if present). One method of executing this task is by learning a coarse approximation of how the ocular region changes from one genuine image to the next, by determining the probability of true deformation through MAP estimation. By maximizing the posterior probability distribution on the latent deformation variables, the prior distribution could be used to improve the results from correlation, and find the proper similarity.

The most likely parameter vector $\mathbf{d}$, that describes the deformations for some (possibly nonlinear) image transformation between the probe image and template, assuming the probe is of the genuine class are determined by the MAP estimation process. As the computational complexity is high (caused by modeling all possible deformations), the deformation is restricted to a parameterized model described by a parameter vector $\mathbf{d}$. This restricts the prior distribution to the specific parameters which are defined on a space with low dimensionality, which are modeled as a multivariate normal distribution. Specifically, $\mathbf{d}$ is defined such that no deformation occurs at the zero vector, which is assumed to be the mean of the distribution, leaving only the covariance to be estimated. To determine the deformation parametrization, a coarse vector field model is used, in which the input image is divided into a set of small regions with corresponding translation vectors $\left\{\left(\Delta x_{i}, \Delta y_{i}\right)\right\}$ and the deformation parameter vector $\mathbf{d}=\left(\triangle x_{1}, \Delta y_{1}, \cdots, \triangle x_{N}, \Delta y_{N}\right)^{t}$. Since the generative probability is defined over a large dimensionality (number of pixels in the probe), parameter estimation can become a daunting task. Thus, the fusion OTSDF output $S(I, T ; \mathbf{d})$ is used as a similarity measure between the image $I$ and the template $T$ at relative deformation $\mathbf{d}$, setting $p(I \mid T, \mathbf{d})=p(S(I, T ; \mathbf{d}))$.

\subsubsection{Score Calculation}

Estimating the posterior distribution of deformation given the observed image, can become computationally expensive, given the number of values the deformation vector can take. Thus, a variant of Pearl's message passing algorithm is used to estimate the marginal posterior distributions at each patch or node in the probabilistic graphical model. Finally, assuming a sparse, acyclic graphical model, a loopy belief propagation model is used to estimate the marginal posterior distribution at each node. The similarity measures from correlation for each image region are multiplied by the marginal posterior distribution of deformation given 
the observed image, $P\left(\mathbf{d}_{k} \mid O\right)$. The final score is considered to be summation of the similarity measures from all the patches in the probe image.

\section{Experiments and Results}

The FOCS database contains images that are acquired from 136 unique subjects. The number of samples per subject varies from 2 to 236 . At least 123 subjects have 10 samples each. In total, the FOCS database contains 9581 images, of which 4792 images correspond to the left eye, and the remaining 4789 correspond to the right eye. For comparison purposes, the recognition performance of iris biometric is also studied. Based on the output obtained from the eye detector, the iris was segmented using a modified version of the active contours without edges technique, originally proposed by Chan and Vese [4]. The segmented iris was encoded and matched using a publicly available, open source iris recognition system [10].

For both the iris and periocular biometrics, matching is performed independently for each of the left and the right side images. For the left-to-left matching, the number of genuine and impostor scores were 267,392 and $22,695,872$, respectively. For the right-to-right matching, these numbers were observed to be 267,273 and $22,667,248$, respectively. To facilitate score level fusion using the sum-rule, the scores obtained from each technique were: (i) independently normalized to a range of $[0,1]$ using the min-max normalization scheme, and (ii) converted to similarity scores. The performance of each individual technique, along with that of the fusion scheme, were evaluated by considering the (a) Equal Error Rate (EER) value, and (b) the value of False Reject Rate (FRR) at a fixed value $(0.1 \%)$ of the False Accept Rate (FAR). Both these values were deduced from the Receiver Operating Characteristic (ROC) curves. The ROC curves for the right-toright matching ${ }^{1}$ using each technique are shown in Figure 6. The normalized histograms of the genuine and impostor scores for right-to-right matching is provided in Figure 7. Table 1 summarizes the performances obtained using each technique for both left-to-left and right-to-right matching. From the results, it can be observed that our initial hypothesis that the periocular biometric would perform better than the iris biometric holds true. Among the various periocular recognition techniques, PDM and modified SIFT provide better performance than $\mathrm{GOH}$. This is because $\mathrm{GOH}$ is a global feature extraction scheme, which can result in good performance only if the two images are perfectly registered or localized. On the other hand, modified SIFT and PDM provide better performance as they consider the keypoint and deformation information, respectively.

\footnotetext{
${ }^{1}$ The ROC curves for the left-to-left matching were observed to be similar to that of right-to-right matching.
}

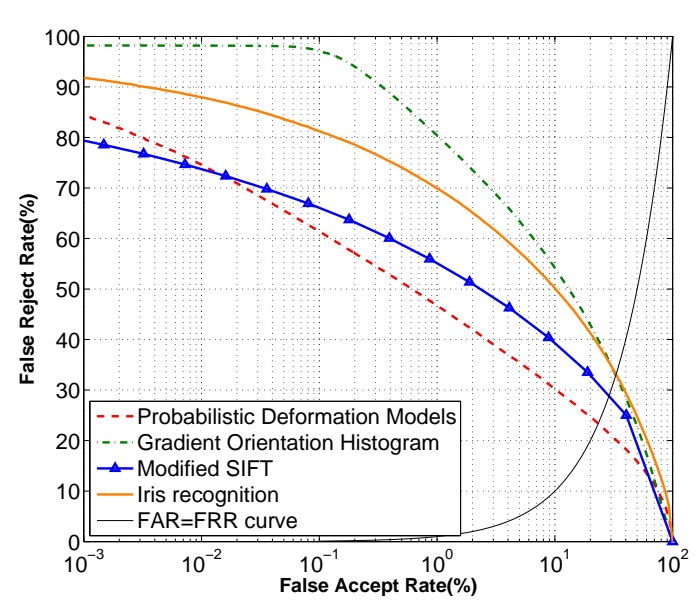

Figure 6. ROC curves for right-to-right matching of FOCS database using the three individual techniques.

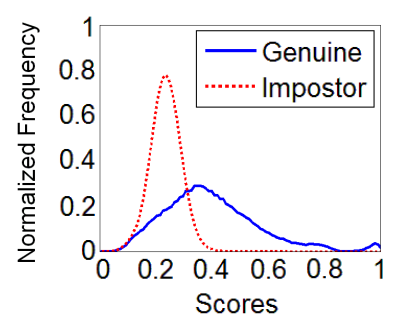

(a)

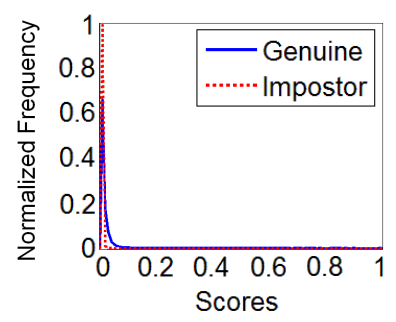

(c)

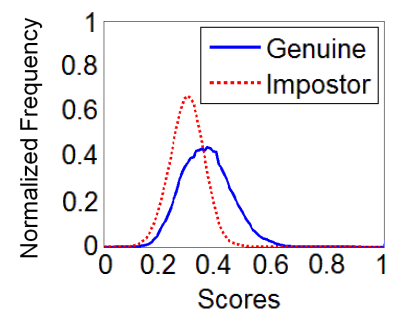

(b)

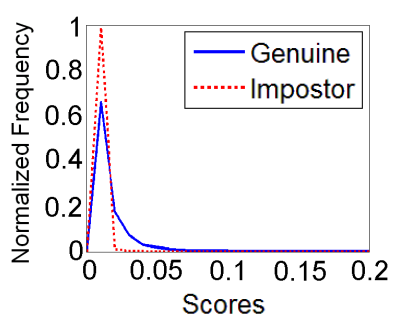

(d)
Figure 7. Normalized genuine and impostor score distributions for right-to-right matching of FOCS database using: (a) Probabilistic Deformation Models, (b) Gradient Orientation Histograms, and (c) modified SIFT. (d) A close up version of the modified SIFT scores.

To observe the performance of each technique with respect to some of the non-ideal factors present in the database, two sets containing 30 images each from the FOCS database were assembled. The images in the first set exhibited non-rigid deformations, while those in the second set contained large variations in illumination levels. The normalized genuine scores obtained by matching these images against the other images of the database, using each of the three techniques are summarized in Figure 8 using 
Table 1. EER values and the values of FRR at a fixed value of FAR $(0.1 \%)$ for left-to-left and right-to-right matching on the FOCS database.

\begin{tabular}{|c|c|c||c|c|}
\hline \multirow{2}{*}{} & \multicolumn{2}{|c||}{ Left-to-left } & \multicolumn{2}{c|}{ Right-to-right } \\
\cline { 2 - 5 } & EER & FRR & EER & FRR \\
\hline PDM & $23.4 \%$ & $58.5 \%$ & $23.9 \%$ & $61.4 \%$ \\
\hline GOH & $32.9 \%$ & $97.4 \%$ & $33.2 \%$ & $97.0 \%$ \\
\hline m-SIFT & $28.8 \%$ & $67.8 \%$ & $27.2 \%$ & $65.9 \%$ \\
\hline Iris & $33.1 \%$ & $81.3 \%$ & $35.2 \%$ & $81.2 \%$ \\
\hline
\end{tabular}

box plots. From the figure, it can be observed that PDM and $\mathrm{GOH}$ provide higher score values for images containing deformations and poor illumination, respectively. This is because the PDM technique measures the correlation between image patches to generate a score, while the GOH technique performs illumination normalization and considers the gradient orientation of the pixels.

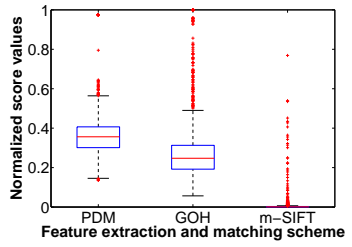

(a)

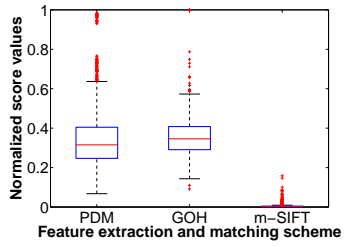

(b)
Figure 8. Box plots for normalized genuine scores corresponding to select images from the FOCS database, containing large variations in (a) deformation and (b) illumination.

A weighted sum rule was used to perform the score level fusion of the three periocular techniques. ${ }^{2}$ The optimal weights for fusion were determined separately to achieve two separate objectives: (i) to minimize the EER and (ii) to minimize the value of FRR at a fixed value $(0.1 \%)$ of FAR. For both the left-to-left and right-to-right matching, the optimal weight $\operatorname{set}^{3}\left[w_{1}, w_{2}, w_{3}\right]$ for objectives (i) and (ii) were observed to be $[0.1,0.1,0.8]$, and $[0.75,0.15,0.10]$, respectively. The ROC curves for the various combinations of fusion for the right-to-right matching are provided in Figure 9. Table 2 summarizes the performances obtained for both left-to-left and right-to-right matching after performing the weighted score-level fusion.

For objective (i), the weight associated with the Modified SIFT matcher was very high $(=0.8)$. On the other hand, for objective (ii), the weight associated with the PDM matcher was very high $(=0.75)$. This suggests the importance of the fusion approach - a single technique cannot be expected to

\footnotetext{
${ }^{2}$ Incorporation of the iris matcher in the fusion scheme degraded matching accuracy. Thus, only the ocular matchers were used in the fusion framework.

${ }^{3}$ The weights $w_{1}, w_{2}$ and $w_{3}$ correspond to PDM, GOH, and m-SIFT, respectively.
}

handle the diversity of images present in the FOCS dataset. By adopting an information fusion scheme, the benefits of individual matchers are highlighted in different portions of the ROC curve.

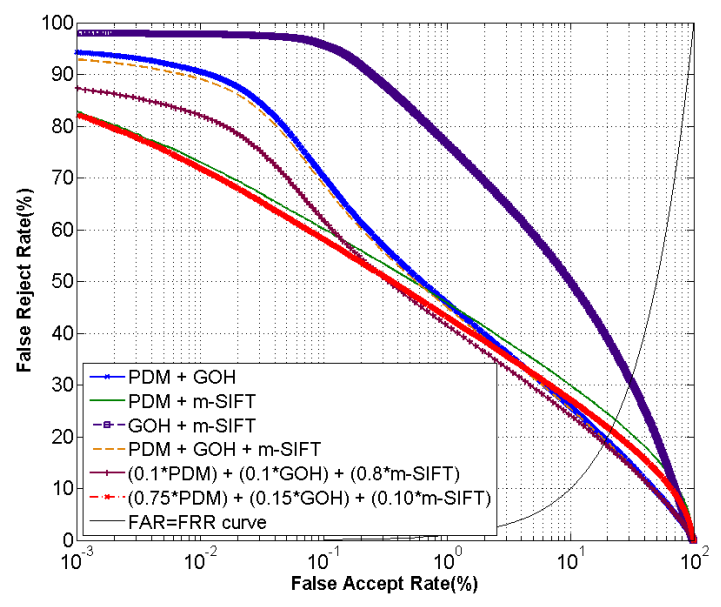

Figure 9. ROC curves after performing fusion for right-to-right matching of the FOCS database.

From Table 2, it can be observed that the fusion of all three techniques provides better performance than fusion of any two individual techniques, thereby suggesting the strength of the proposed approach. It has to be noted that the performances reflect the objectives that are used for obtaining the optimal weights. The optimal weights for fusion in high security applications could be determined by minimizing the FRR value at a fixed FAR. On the other hand, if the focus is more on user convenience, the optimal weights could be determined by minimizing the EER values.

To further evaluate its performance, the proposed fusion approach was applied on a subset of ocular images that were gathered from the Face Recognition Grand Challenge (FRGC) Database. A set of 1136 left and 1136 right ocular images were used for this purpose, corresponding to a total of 568 unique subjects (2 samples per eye per subject). The first image of every subject was used as a gallery entry, while the other was used as the probe. This experimental setup was used in [13], and helps us compare the performance of the proposed approach with that of [13] ${ }^{4}$. The results are listed in Table 3 . From the table it can be observed that m-SIFT provides the best recognition performance compared to PDM and GOH. Score level fusion of $\mathrm{m}$-SIFT and PDM provides the lowest EER (e.g., $1.59 \%$ for left-to-left matching), confirming the significance of a fusion approach over a single technique for ocular matching.

\footnotetext{
${ }^{4}$ The lowest EER reported in [13] was $6.96 \%$. This value was obtained by the score level fusion of left-to-left with right-to-right matching using the standard implementation of SIFT.
} 
Table 2. EER values and FRR values at a fixed value of FAR (0.1\%) for both left-to-left and right-to-right matching of the FOCS database, after performing weighted score-level fusion.

\begin{tabular}{|c|c|c||c|l|}
\hline \multirow{2}{*}{} & \multicolumn{2}{|c||}{ Left-to-left } & \multicolumn{2}{c|}{ Right-to-right } \\
\cline { 2 - 5 } & EER & FRR & EER & FRR \\
\hline PDM+GOH & $19.5 \%$ & $71.7 \%$ & $19.4 \%$ & $70.1 \%$ \\
\hline PDM+m-SIFT & $23.9 \%$ & $57.6 \%$ & $23.3 \%$ & $60.0 \%$ \\
\hline GOH+m-SIFT & $31.2 \%$ & $96.2 \%$ & $27.2 \%$ & $95.5 \%$ \\
\hline PDM+GOH+m-SIFT & $19.3 \%$ & $70.5 \%$ & $19.3 \%$ & $68.8 \%$ \\
\hline$(0.1 * \mathrm{PDM})+(0.1 * \mathrm{GOH})+(0.8 * \mathrm{~m}-\mathrm{SIFT})$ & $\mathbf{1 8 . 8 \%}$ & $63.8 \%$ & $\mathbf{1 8 . 8 \%}$ & $61.4 \%$ \\
\hline$\left(0.75^{*} \mathrm{PDM}\right)+\left(0.15^{*} \mathrm{GOH}\right)+(0.10 * \mathrm{~m}-\mathrm{SIFT})$ & $21.7 \%$ & $\mathbf{5 5 . 4 \%}$ & $21.2 \%$ & $\mathbf{5 8 . 0 \%}$ \\
\hline
\end{tabular}

Table 3. EER values for left-to-left and right-to-right matching on a subset of the FRGC database.

\begin{tabular}{|c|c|c|}
\hline & Left-to-Left & Right-to-Right \\
\hline PDM & $4.36 \%$ & $3.84 \%$ \\
\hline GOH & $19.74 \%$ & $18.61 \%$ \\
\hline m-SIFT & $2.48 \%$ & $2.37 \%$ \\
\hline$(0.3 *$ PDM $)+(0.7 * m-S I F T)$ & $\mathbf{1 . 5 9 \%}$ & $\mathbf{1 . 9 3 \%}$ \\
\hline
\end{tabular}

\section{Conclusions and Future work}

This work presents an information fusion approach to perform ocular recognition on highly non-ideal images in which iris recognition may not be feasible. Since ocular recognition uses the information surrounding the eye, the problem of iris segmentation is automatically avoided. The proposed approach combines the biometric information extracted using three different techniques (Probabilistic Deformation Models, Gradient Orientation Histogram, and modified SIFT) at the score-level using the sum rule. The three techniques help in consolidating global, local and deformation information pertaining to the images. When the performance of iris recognition is compared with that of the proposed fusion approach, the EER is reduced from $33.1 \%$ to $18.8 \%$ for the left-side images and from $35.2 \%$ to $18.8 \%$ for the right-side images, respectively. These error rates reflect the best matching performance for ocular recognition using a challenging database [3]. Further, experiments on a subset of the FRGC database confirms the effectiveness of the proposed fusion approach. Future work would include the incorporation of other techniques (e.g., bag of features, patch based deformation models, etc.) for feature extraction and matching. Further, we are exploring the design of an adaptive fusion scheme, in which the weights for the individual matchers will be assigned based on the quality of the input images.

\section{References}

[1] C. Belcher and Y. Du. Region-based SIFT approach to iris recognition. Optics and Lasers in Engineering, 47(1):139-147, 2009. 4
[2] S. Bharadwaj, H. Bhatt, M. Vatsa, and R. Singh. Periocular biometrics: When iris recognition fails. In BTAS, Sep. 2010. 1, 3

[3] V. Boddeti, J. M. Smereka, and B. V. K. Vijaya Kumar. A comparative evaluation of iris and ocular recognition methods on challenging ocular images. In $I J C B$, Oct. 2011. 8

[4] T. Chan and L. Vese. Active contours without edges. IEEE TIP, 10(2):266-277, Feb. 2001. 6

[5] N. Dalal, B. Triggs, and C. Schmid. Human detection using oriented histograms of flow and appearance. In ECCV, volume 2, pages 428441, 2006. 3

[6] J. Daugman. High confidence visual recognition of persons by a test of statistical independence. IEEE TPAMI, 15(11):1148-1161, 1993. 1

[7] D. G. Lowe. Object recognition from local scale-invariant features (SIFT). In ICCV, pages 1150-1157, 1999. 3, 4

[8] D. G. Lowe. Distinctive image features from scale-invariant keypoints. IJCV, 60:91-110, Nov. 2004. 3

[9] J. Luo, Y. Ma, M. Kawade, and B. Lu. Person-specific SIFT features for face recognition. In ICASSP, pages 223-228, Jun. 2007. 4

[10] L. Masek and P. Kovesi. MATLAB source code for a biometric identification system based on iris patterns. The University of Western Australia, 2003. 6

[11] P. Miller, J. Lyle, S. Pundlik, and D. Woodard. Performance evaluation of local appearance based periocular recognition. In BTAS, pages 1-6, Sep. 2010. 1, 3

[12] NIST. Face and Ocular Challenge Series database. http://www.nist.gov/itl/iad/ig/focs.cfm. 2

[13] U. Park, R. Jillela, A. Ross, and A. K. Jain. Periocular biometrics in the visible spectrum. IEEE TIFS, 6(1):96-106, Mar. 2011. 1, 7

[14] U. Park, A. Ross, and A. K. Jain. Periocular biometrics in the visible spectrum: A feasibility study. In BTAS, pages 1-6, Sep. 2009. 1, 3

[15] V. P. Pauca, M. Forkin, X. Xu, R. Plemmons, and A. Ross. Challenging ocular image recognition. In BTHI, SPIE, volume 8029, pages 80291V-1-80291V-13, Apr. 2011. 3, 4

[16] P. Réfrégier. Filter design for optical pattern recognition: multicriteria optimization approach. Journal of Optics Letters, 15(15):854856, 1990. 4

[17] J. Thornton, M. Savvides, and B. V. K. Vijaya Kumar. A Bayesian approach to deformed pattern matching of iris images. IEEE TPAMI, pages 596-606, 2007. 3, 4

[18] A. Vedaldi and B. Fulkerson. VLFeat: An open and portable library of computer vision algorithms, available at http://www.vlfeat.org/, 2008. 4

[19] B. V. K. Vijaya Kumar, M. Savvides, C. Xie, K. Venkataramani, J. Thornton, and A. Mahalanobis. Biometric verification with correlation filters. Journal of Applied Optics, 43(2):391-402, Jan. 2004. 3

[20] D. Woodard, S. Pundlik, P. Miller, R. Jillela, and A. Ross. On the fusion of periocular and iris biometrics in non-ideal imagery. In ICPR, pages 201-204, Aug. 2010. 1 